\title{
HEAD-ON COLLISIONS OF TWO SOLITARY WAVES WITH DIFFERENT AMPLITUDES USING IMAGING TECHNIQUES
}

\begin{abstract}
Motohiko Umeyama ${ }^{1}$
The behavior of two unequal solitary waves during head-on collision was experimentally studied by optical and particle-tracer methods. Spatial surface profiles were measured using the particle mask correlation method and the image thresholding method, which detects the air-water boundary as a set of locally extreme luminance values. The measured surface displacement of the colliding wave was compared with the corresponding shape of a third-order perturbation approximation. In addition, to estimate the phase shift from the crest at an arbitrary point, the instantaneous surface variations were measured by two wave gauges. The kinetic features of the target and the oncoming and colliding waves were measured by a particle image velocimetry method. To solve the phase shift, we acquired the velocity fields of the colliding waves in a series of experiments and could show our technological advantage over others.
\end{abstract}

Keywords: Solitary wave; Head-on Collision; PIV; Run-up; Phase shifts/

\section{INTRODUCTION}

The "Committee on Waves," set up by the British Association for the Advancement of Science, appointed John Scott-Russell and John Robison (1937) to conduct surface wave experiments in a long wave tank. In 1837, the researchers published a significant report on their findings. Russell (1844) continued his laboratory observations, mainly on the nature of solitary waves, and made an important discovery that the wave speed satisfies $c=\sqrt{g(h+a)}$, where $g$ is the gravitational acceleration, $h$ is the undisturbed water depth, and $a$ is the wave amplitude. Boussinesq (1871) and Rayleigh (1976) mathematically confirmed Russell's empirical formula. They also showed that, for waves of sufficiently small amplitude, the water surface profile follows a $\sec h^{2}$ function. Modifying Rayleigh's method, Korteweg and de Vries (1895) provided the exact solution of oscillatory waves of finite height and finally obtained the solitary wave solution at limited depth. The Korteweg-de Vries (KdV) equation applies not only to the propagation of single solitary waves but also to rear-end collisions of two solitary waves. In the latter case, two solitary waves move at different speeds in the same direction, and the taller wave overtakes the other, surpassing it as time increases. If these waves satisfy the linear superposition principle, they retain their identities after the interaction. However, as reported in Drazin and Johnson (1989), Johnson (1997), and Constantin (2011), two waves traveling at constant speeds interact nonlinearly, inducing a phase shift from their estimated arrival positions.

The discovery of recurrence phenomena in discrete linear systems (1955) triggered great interest in the KdV equation during the latter half of the 20th century. Zabusky and Kruskal (1965) formulated a numerical model of the KdV equation for a pair of unequal solitary waves and reported nonlinear interaction between the waves. Their numerical solutions showed that multiple solitary waves retain their identity, but undergo a phase shift after a collision. Since Gardner et al. (1967) published their analytical solution of the $\mathrm{KdV}$ equation, the elegant theories and unique properties of solitary wave interactions have intrigued mathematicians, physicists, and engineers. However, the analytical method of Gardner et al. is not applicable to solitary waves traveling in different directions because the KdV equation approximates simple wave motions under the dispersion effect. Byatt-Smith (1971) obtained a second-order analytical solution for the nonlinear interaction between a solitary wave and a vertical wall. The Boussinesq equation solves the combined motion of incident and reflected solitary waves, each of which satisfies an appropriate $\mathrm{KdV}$ equation. The second-order solution includes an interaction term that separates the two solitary-wave terms into functions of their respective phase variables. Miles (1977) theoretically investigated the nonlinear interaction between counter-propagating dual solitary waves, decomposing the interaction term into transient and phase-shift terms. Su and Mirie (1980) and Mirie and Su (1982) derived perturbation and numerical solutions of higher-order Boussinesq equations. Their third-order perturbation solution showed that when two solitary waves collide head-on, the profile becomes asymmetric during the interaction, but gradually stabilizes with a phase shift. A third-order dispersive tail also appeared, although the wave amplitudes were unchanged. The predicted

\footnotetext{
${ }^{1}$ Department of Civil \& Environmental Engineering, Tokyo Metropolitan University, 1-1 Minami-ohsawa, Hachioji, Tokyo 192-0397, Japan
} 
dispersive tail, but not the amplitude invariance, was confirmed in numerical studies. Based on inverse scattering, Byatt-Smith $(1988,1989)$ derived coupled perturbed KdV equations for a solitary wave and its reflection from a vertical wall, and the head-on collision of two solitary waves of unequal amplitudes.

Maxworthy (1976) experimentally investigated solitary wave reflections and head-on collisions between two solitary waves in a short flume. The dynamics were recorded by an $8 \mathrm{~mm}$ video recorder at 64 fps. Maxworthy considered two wave-generating methods. First, solitary waves were generated by pulling a flat plate and reflecting the waves from a plane vertical wall; second, multiple waves were generated by opening movable partitions installed at the fronts of small water tanks at both ends of the flume. Maxworthy found that the magnitude of the phase shift was independent of the wave amplitude, and that the maximum run-up height during reflection at the vertical wall was more than twice the initial wave amplitude. Byatt-Smith's theory and Maxworthy's results differed only in the magnitude of the phase shift. Renouard et al. (1985) experimentally reflected solitary waves, and found a transient loss of amplitude in the reflected wave, which recovered over time. They attributed this behavior to the dispersive tail behind the solitary wave. Since then, various solitary waves and their interactions have been experimentally confirmed by researchers such as Hammack et al. (2004), Craig et al. (2006), and Chen and Yeh (2014).

Modern imaging techniques have rapidly progressed the field of fluid mechanics. Significant developments in particle image velocimetry (PIV) and particle tracking velocimetry (PTV) have enabled the visualization of velocity fields and particle paths. Using a PIV system, Umeyama et al. (2010) and Umeyama $(2011,2012)$ measured the water particle velocity and trajectory of surface Stokes waves with and without a steady current. Recently, Umeyama (2016) estimated the velocities and trajectories of water particles, and the velocity vector fields, using a superresolution PIV based on Kalman filtering and $x^{2}$-testing (2000). In the present paper, we investigate the head-on collision of two solitary waves with different amplitudes, focusing on their phase shifts after the interaction. The water surface displacements were measured in two ways: (i) by combining particle mask correlation (PMC) and image thresholding in a smaller test-section; and (ii) by wave-gauge measurements in a larger test-section. The kinematics of the colliding wave were also investigated using a new PIV system, which improves the spatial resolution of the precedent set by Umeyama et al. (2014).

\section{THEORIES}

Consider a cross-section of a wave field perpendicular to the crest line. The system is configured in Cartesian coordinates $(x, z)$, where the $x$-axis is the direction of wave propagation and the $z$-axis points vertically upward. The origin lies on the flat bottom. In an incompressible, inviscid fluid with no surface tension, the surface of a single solitary wave is given by [Boussinesq (1871), Rayleigh (1876)]

$\eta=a \sec h^{2} \sqrt{\frac{3}{4} \frac{a}{h^{3}}}(x-c t) \quad$,

where $c$ is the wave celerity, defined as

$c=\sqrt{g(h+a)}$.

For a head-on collision of two solitary waves, Su and Mirie (1980) derived a third-order solitary-wave solution using the perturbation method. They expressed the surface elevation as

$$
\begin{aligned}
\frac{\eta(x, t)}{h} & =\varepsilon_{R}\left(\zeta_{R}+\frac{3}{4} \varepsilon_{R}\left(\zeta_{R}^{2}-\zeta_{R}\right)+\varepsilon_{R}^{2}\left(\frac{101}{80} \zeta_{R}^{3}-\frac{151}{80} \zeta_{R}^{2}+\frac{5}{8} \zeta_{R}\right)\right) \\
& +\varepsilon_{L}\left(\zeta_{L}+\frac{3}{4} \varepsilon_{L}\left(\zeta_{L}^{2}-\zeta_{L}\right)+\varepsilon_{L}^{2}\left(\frac{101}{80} \zeta_{L}^{3}-\frac{151}{80} \zeta_{L}^{2}+\frac{5}{8} \zeta_{L}\right)\right) \\
& +\varepsilon_{R} \varepsilon_{L}\left(\frac{1}{2}+\left(\frac{7}{4}\left(\varepsilon_{R} \zeta_{R}+\varepsilon_{L} \zeta_{L}\right)-\frac{11}{8}\left(\varepsilon_{R}+\varepsilon_{L}\right)\right)\right) \zeta_{R} \zeta_{L}
\end{aligned}
$$

where $\zeta_{R}$ and $\zeta_{L}$ are the nondimensional right-running and left-running wave profiles, respectively; and $\varepsilon_{R}=a_{R} / h, \varepsilon_{L}=a_{L} / h$. The profiles are given by

$$
\zeta_{R}=\frac{\eta_{R}}{a_{R}}=\operatorname{sech}^{2}\left(\left(\frac{3 \varepsilon_{R}}{4 h^{2}}\right)^{\frac{1}{2}}\left(1-\frac{5}{8} \varepsilon_{R}+\frac{71}{128} \varepsilon_{R}^{2}\right)\left(x-c_{R} t+\theta_{R}\right)\right)
$$


$\zeta_{L}=\frac{\eta_{L}}{a_{L}}=\operatorname{sech}^{2}\left(\left(\frac{3 \varepsilon_{L}}{4 h^{2}}\right)^{\frac{1}{2}}\left(1-\frac{5}{8} \varepsilon_{L}+\frac{71}{128} \varepsilon_{L}^{2}\right)\left(x+c_{L} t+\theta_{L}\right)\right)$,

where $a_{R}, c_{R}$, and $\theta_{R}$ denote the amplitude, celerity, and phase shift, respectively, of the right-running wave, and $a_{L}, c_{L}$, and $\theta_{L}$ are the corresponding variables for the left-running wave. The phase shift is defined as the location difference between the crest after the collision and the crest of a single solitary wave simultaneously traveling without interaction. The celerity and phase shift of the right-running wave are calculated as

$$
\begin{aligned}
& c_{R}=c\left(1+\frac{\varepsilon_{R}}{2}-\frac{3}{20} \varepsilon_{R}^{2}+\frac{3}{56} \varepsilon_{R}^{3}\right) \\
& \theta_{R}=h\left(\frac{\varepsilon_{L}}{3}\right)^{\frac{1}{2}}\left(1+\frac{\varepsilon_{L}}{8}+\frac{3}{4} \varepsilon_{R}\right)
\end{aligned}
$$

Similar formulas for the left-running wave are

$$
\begin{aligned}
& c_{L}=c\left(1+\frac{\varepsilon_{L}}{2}-\frac{3}{20} \varepsilon_{L}^{2}+\frac{3}{56} \varepsilon_{L}^{3}\right) \\
& \theta_{L}=-h\left(\frac{\varepsilon_{R}}{3}\right)^{\frac{1}{2}}\left(1+\frac{\varepsilon_{R}}{8}+\frac{3}{4} \varepsilon_{L}\right)
\end{aligned}
$$

To find the maximum run-up, we specify $x-c_{R} t+\theta_{R}=0$ in Eq. (4) and $x-c_{L} t+\theta_{L}=0$ in Eq. (5) and insert the resulting $\zeta_{R}$ and $\zeta_{L}$ into Eq. (3). This gives

$\frac{\eta_{\max }}{h}=\varepsilon_{R}+\varepsilon_{L}+\frac{1}{2} \varepsilon_{R} \varepsilon_{L}\left(1+\frac{3}{4}\left(\varepsilon_{R}+\varepsilon_{L}\right)\right)$

where $\eta_{\max }$ is the maximum run-up elevation.

\section{EXPERIMENTS}

Experiments were performed in a wave tank of length $25.0 \mathrm{~m}$, width $0.8 \mathrm{~m}$, and depth $1.0 \mathrm{~m}$. The wave-generating system consisted of a servo-controlling device and a steel vertical plate that generated a solitary wave at one end of the tank. The opposite end was outfitted with a wooden vertical wall for reflecting the incident wave. The paddle movement was controlled by a programmed electrical signal input from an arbitrary waveform synthesizer. This system generates highly accurate solitary waves. Single solitary waves can be generated as [Goring, (1979)]

$\xi(t)=A \tanh 7.6\left(\frac{t}{\tau}-\frac{1}{2}\right)$ where $A=4 \sqrt{\frac{a h}{3}}$, and $\tau=\frac{4}{\sqrt{g(h+a)}} \sqrt{\frac{h^{3}}{3 a}}\left(\tanh ^{-1}(0.999)+\frac{a}{h}\right)$

In this expression, A denotes the paddle stroke and $\tau$ is the duration of motion. Following this precedent, we reproduce the surface profile using the tanh signal. For colliding counter-propagating solitary waves, Umeyama et al. (2014) proposed the following paddle trajectory:

$$
\begin{aligned}
\xi(t)=\frac{A}{2} \tanh \left(2 \pi\left(\frac{t}{\tau}-\frac{1}{2}\right)\right)+\frac{A}{2} \tanh \pi & 0 \leq t \leq \tau \\
A \tanh \pi & t \geq \tau
\end{aligned}
$$

In generating dual solitary waves, we must prescribe the periods of the first and second generated waves and the stationary interval. When the first wave reaches the vertical wall, it is reflected and propagated backward, generating the second wave. The first and second waves collide head-on near the center of the wave tank. The water surface data (detailed in the latter half of this section) were obtained from two sets of experiments. In the first set, the surface displacements were visually measured by a laser and a CCD camera. The test section was located between 14.0 and $15.0 \mathrm{~m}$ from the initial (stationary) position of the paddle. The second set of experiments investigated the phase shift induced by the head-on collision using two wave gauges. The test section was located 12.5 to $16.5 \mathrm{~m}$ downstream of the paddle.

The instantaneous velocity field during the collision of dual solitary waves was measured by a PIV system. The PIV comprised an 8-W frequency-doubled Nd:YAG laser and a high-speed CCD camera with a resolution of 1,024 x 1,024 pixels, a maximum frame rate of 500,000 fps, and a maximum shutter speed of $1 / 1,000,000$. A visualization probe was connected to the laser head by an optical fiber and set on a beam above the wave tank. A 2-mm thick laser sheet was directed toward the 
water surface. The area coverage of the CCD camera was up to $80 \mathrm{~cm} \times 15 \mathrm{~cm}$, corresponding to a resolution of 1,024 x 192 pixels. The frame rate was $250 \mathrm{fps}$, and the shutter speed was $1 / 700$. The tank water was seeded with DIAION, with a grain size and specific density of $0.25 \mathrm{~mm}$ and 1.01 , respectively.

The temporal and spatial variations of the water surface were observed by combining PMC with the image thresholding method. The PMC method identifies the individual tracer particles suspended below the water surface by a pattern matching procedure using a template, such as twodimensional Gaussian brightness distributions (Chambarelet al., 2009). Every particle in the recorded image displays a characteristic brightness pattern that peaks near the center and steadily decreases in the radial direction. In the image pre-processing, the recorded frames were scanned pixel-by-pixel to find occurrences of this pattern, and the air-water boundary was estimated from the seeded particles in each recorded image. The spatial distribution of the water surface at each instant was estimated from the discontinuous edge positions. The imaging process was advanced to the next stage using the image thresholding method, which was originally designed to reduce a grayscale image to a binary image [Umeyama (2008)]. Image thresholding performs an easy and convenient segmentation based on the intensity differences between the foreground and background regions of an image. Among the numerous techniques available, we employed a general boundary-detection method called edge-based thresholding. In this approach, an edge is detected as an abrupt change in the luminance value. Under illumination by the laser sheet, the air-water surface was detected as the region of maximum intensity. After the combined PMC/image thresholding analysis, the processed data were transformed to realscale data in the orthographic plane.

The experimental conditions are summarized in Table 1. In a series of head-on collision tests, the paddle motion was controlled to provide two consecutive solitary waves of different amplitudes. The quiescent water depth was maintained at $7.5 \mathrm{~cm}$. In cases $\mathrm{V} 1-\mathrm{V} 8$, the kinematic changes accompanying the head-on collision were investigated by measuring the velocity components and spatial surface displacements by the two-dimensional PIV in the test section. In addition to these imaging methods, the surface variations were recorded at two selected sites separated by $388 \mathrm{~cm}$ in the test section. The left and right gauges (GAUGE-1 and GAUGE-2) were stationed on the reflected-wall and wavemaker sides, respectively. In cases G1-G6, the amplitude of the left-running wave was maintained constant while that of the right-running wave was altered; in cases G7-G16, the tests were repeated for a new amplitude of the left-running wave. The data were measured by resistance-type probes. The measurement accuracy was determined by the sampling frequency $(47 \mathrm{~Hz})$.

\begin{tabular}{|c|c|c|c|c|c|c|c|c|}
\hline \multicolumn{7}{|c|}{ Table 1 Experimental Conditions. ( unit : cm ) } \\
\hline Cases & G1 & G2 & G3 & G4 & G5 & G6 & G7 & G8 \\
\hline$a_{R}$ & 1.13 & 1.23 & 1.36 & 1.48 & 1.64 & 1.74 & 1.29 & 1.39 \\
\hline$a_{L}$ & 2.00 & 2.02 & 2.01 & 2.09 & 2.11 & 2.15 & 1.50 & 1.51 \\
\hline Cases & G9 & G10 & G11 & G12 & G13 & G14 & G15 & G16 \\
\hline$a_{R}$ & 1.50 & 1.60 & 1.82 & 1.91 & 2.02 & 2.08 & 2.35 & 2.43 \\
\hline$a_{L}$ & 1.51 & 1.51 & 1.57 & 1.53 & 1.56 & 1.56 & 1.55 & 1.60 \\
\hline Cases & V1 & V2 & V3 & V4 & V5 & V6 & V7 & V8 \\
\hline$a_{R}$ & 1.00 & 1.19 & 1.47 & 1.58 & 1.27 & 1.61 & 2.03 & 2.59 \\
\hline$a_{L}$ & 2.11 & 2.13 & 2.09 & 2.10 & 1.41 & 1.41 & 1.41 & 1.41 \\
\hline$\eta_{\max }$ & 3.44 & 3.66 & 3.90 & 4.29 & 2.92 & 3.23 & 3.50 & 4.20 \\
\hline
\end{tabular}

\section{RESULTS}

Fig. 1 shows the spatial surface profiles during four head-on collision tests: (a) V1, (b) V3, (c) V5, and (d) V7, acquired by the combined PMC and image thresholding methods. The fifteen pivotal moments during the dual solitary-wave interaction are presented in order of time. Each panel includes three surface profiles. The cross, triangular, and circular symbols indicate the right-running, leftrunning, and colliding wave profiles, respectively. The right-running wave moves from left to right after reflection from the vertical wall, while the left-running wave is directly sourced from the 

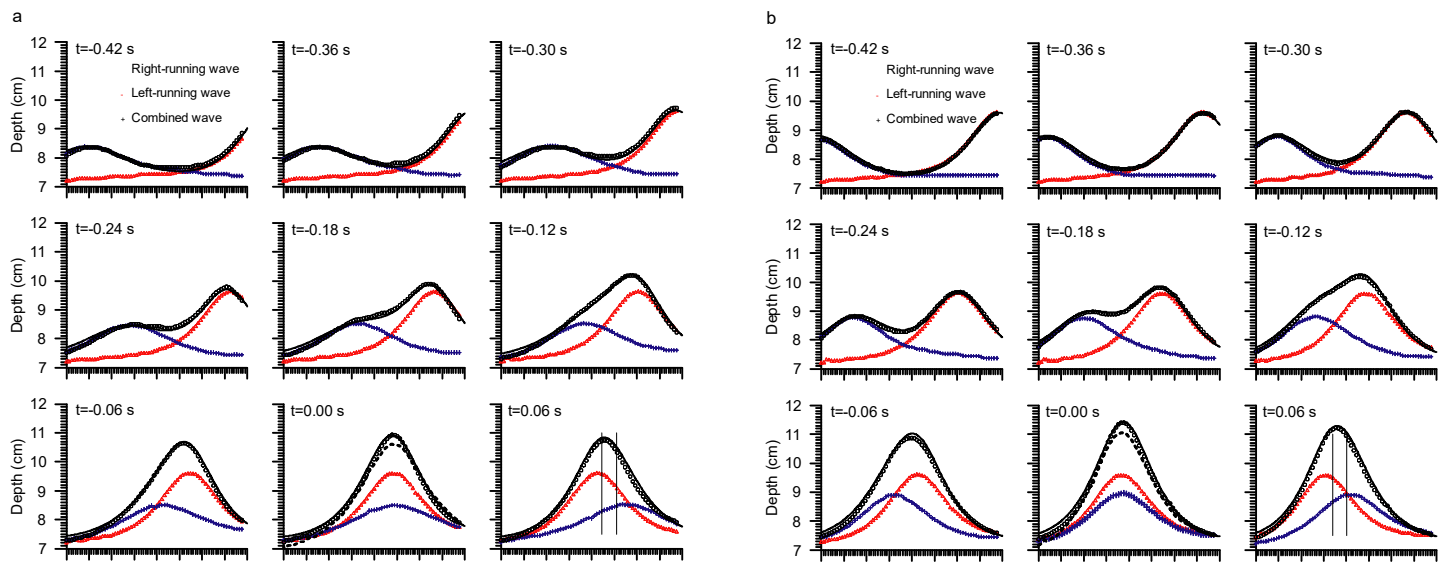

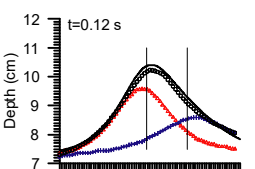
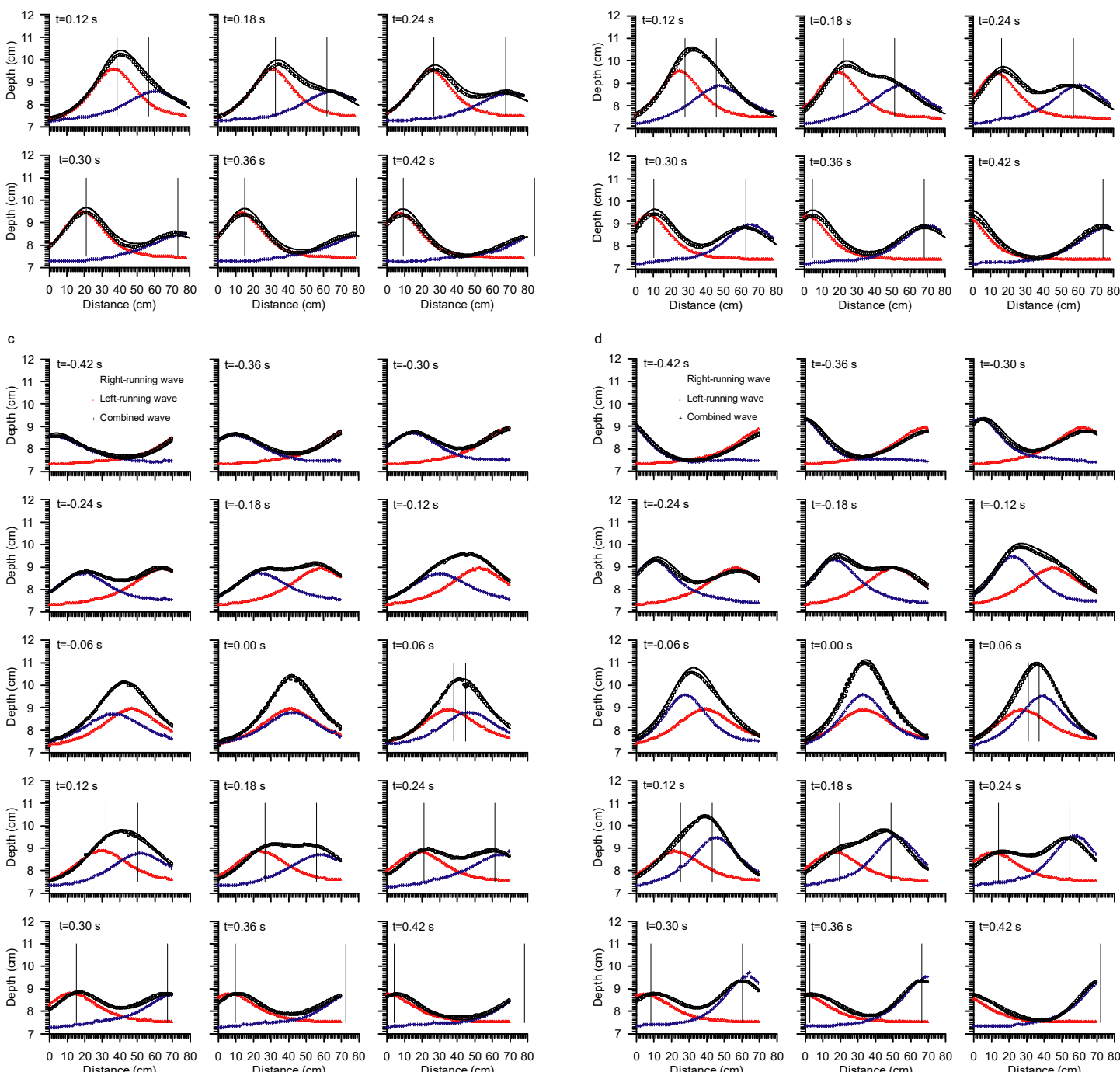

Fig.1 Experimental water surface displacements and colliding wave profiles for a third-order perturbation approximation for (a) V1, (b) V3, (c) V5, and (d) V7.

wavemaker. The temporal origin $(t=0.0 \mathrm{~s})$ is decided as the instant at which the water surface of the colliding waves is maximally elevated. The preceding and following time domains are denoted as positive and negative, respectively. Before the colliding wave is created in front of the CCD camera (which covers $90 \mathrm{~cm} \times 73 \mathrm{~cm}$ ), the interaction has already elevated the water surface before $t=-4.2 \mathrm{~s}$. However, the surface profile of the colliding wave is almost consistent with linear superposition of the 


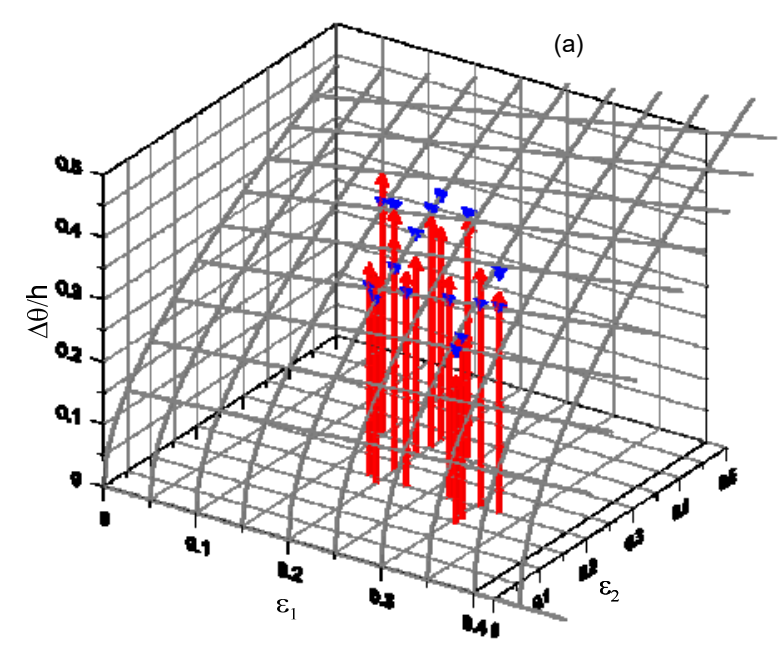

(b)

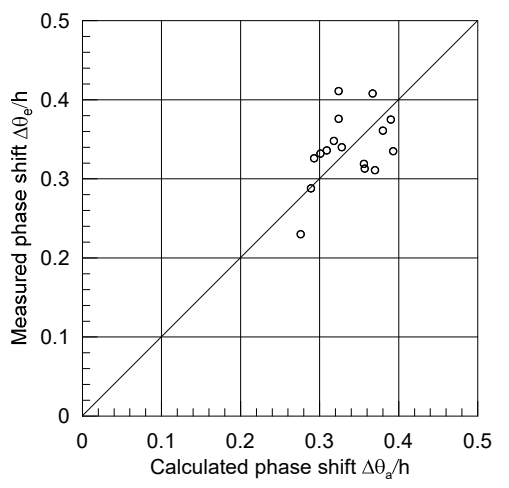

Fig.2 (a) Phase shift against target and oncoming wave amplitudes.

(b) Comparisons between measured and calculated phase shifts.

right- and left-running solitary waves. From -0.36 to $0.00 \mathrm{~s}$, the surface gradually rose while both wave crests were preparing to meet each other. Over time, the single solitary waves merged into a single wave, and their crests gradually disappeared from the surface of the compound wave. To examine the effect of the nonlinear interaction on the maximum run-up elevation at $t=0.0 \mathrm{~s}$, we plotted the superimposed left- and right-running wave profiles (dotted curve in Fig. 1). The theoretical surface elevation was obviously higher than the summed incoming waves. After the meeting of two solitary-wave crests, the surface returned to its original state from $t=0.06 \mathrm{~s}$ to $t=0.42 \mathrm{~s}$. Meanwhile, the compound wave separated into two solitary waves with respective phase shifts. For comparison between the measured and calculated surface variations, the theoretical solutions of $\mathrm{Su}$ and Mirie [14] are plotted as solid lines in 16 instances. Although the experimental and theoretical surface features match in the preceding time domain, the measured amplitude is smaller than the estimated amplitude in the following time domain. The amplitude attenuation becomes more conspicuous as the amplitudes of the oncoming and target waves diverge. To clarify the crest delay, we inserted two vertical solid lines in the panels of positive $t$, indicating the theoretical crest positions of the right- and left-running waves. The compound wave appeared to propagate with a slightly lower celerity than the single solitary waves. Around the corresponding crests, the right- and left-running waves displayed steeper profiles than the compound wave. The combined PMC and image thresholding clearly revealed the phase shifts, despite the limited measurement area. The measured phase shifts can be assessed by relating the target-wave amplitude $\left(a_{1}\right)$ to the oncoming-wave amplitude $\left(a_{2}\right)$ in a three-dimensional Cartesian coordinate system. Fig. 2 (a), the $x$ - and $y$-axes are the dimensionless wave amplitudes $\varepsilon_{1}=a_{1} / h$ and $\varepsilon_{2}=a_{2} / h$, respectively, and the $z$-axis is the dimensionless phase shift $\Delta \theta / h$. The curves on the theoretical three-dimensional phase-shift diagram are obtained from Su and Mirie's (1980) solution. The bar chart presents the measured phase-shift data, and the inverted triangles are the theoretical values for each experimental parameter set. Theoretically, the phase shift of the target wave should increase with increasing amplitude of the oncoming wave, but this expectation is not supported by the experimental results. Before making a conclusive decision, we require more data for a statistical analysis. At this stage, we infer only that the phase shift results from changes in any two variables. Under the present experimental conditions $\left(0.14<\varepsilon_{1}, \varepsilon_{2}<0.33\right)$, the

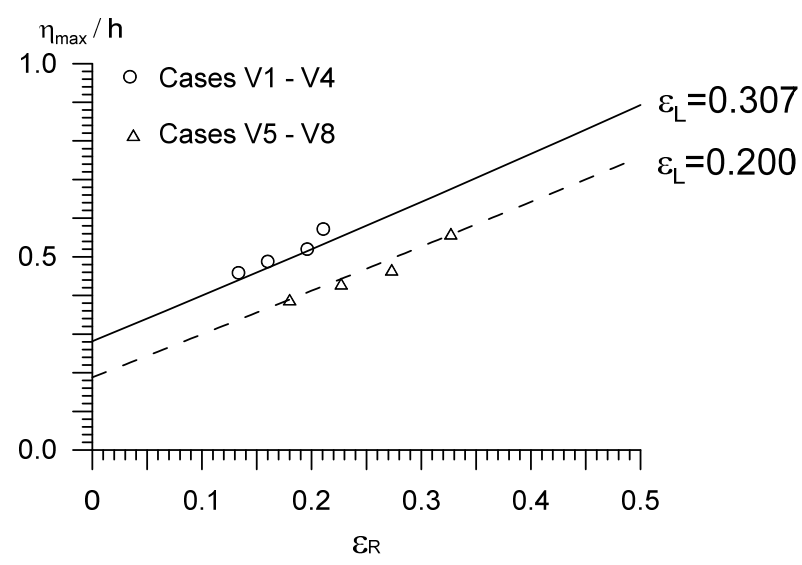

Fig. 3 Maximum elevation vs. relative amplitudes. 

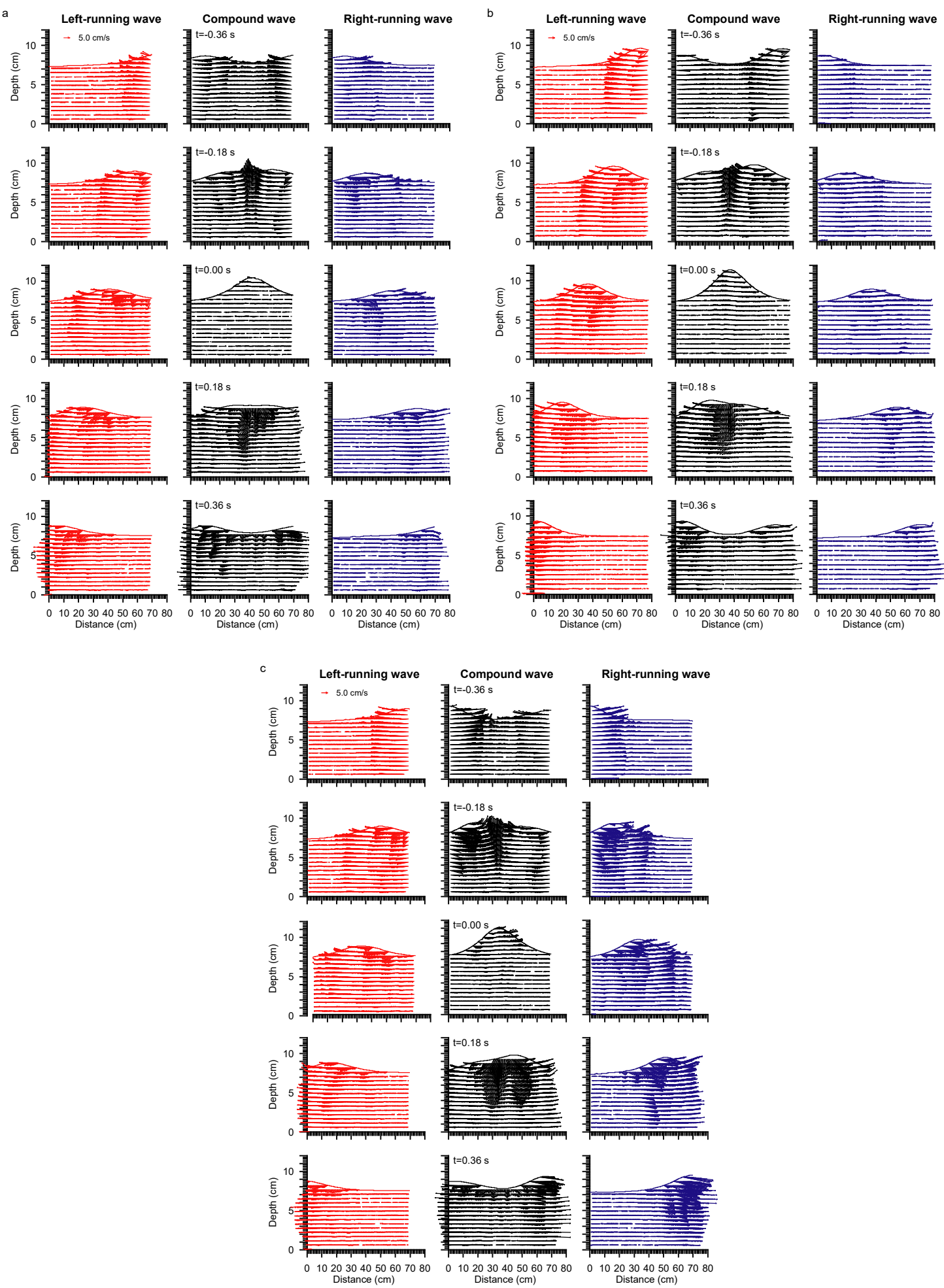

Fig. 4 Experimental velocity fields for right-running, left-running, and compound waves: (a) V5, (b) V3, and (c) V7.

measured phase shift ranged slightly more widely $\left(0.23<\Delta \theta_{e} / h<0.41\right)$ than the calculated phase shift $\left(0.27<\Delta \theta_{a} / h<0.39\right)$. To illustrate this subtle difference, we compare the measured and computed phase shifts in Fig. 2 (b). The dimensionless measured phase shift $\left(\Delta \theta_{e} / h\right)$ agrees with the calculated one $\left(\Delta \theta_{a} / h\right)$ within the error margins, although the error in the measurement is large (up to $\sim 10 \%$ ). 

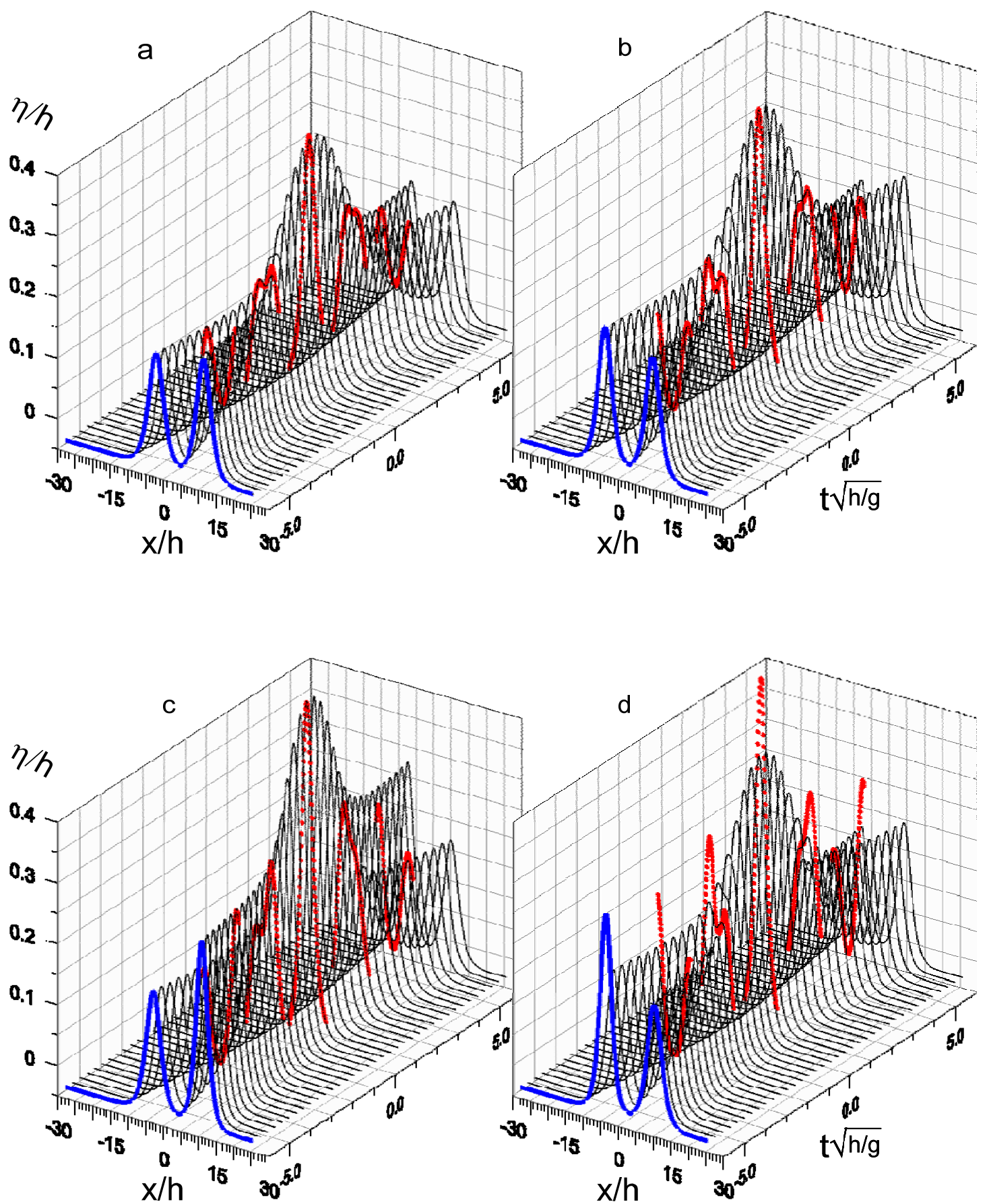

Fig. 5 Asymmetric head-on collision of two solitary waves of different amplitudes for (a) V5, (b) V6, (c) V3, and (d) V8.

Given the unreliable determination of the crest locations, this error is probably reasonable. As the positions of the crests on the compound wave surface rapidly altered after $t=0.0 \mathrm{~s}$, they were difficult to determine in practice. The measured and predicted phase shifts were consistent within the experimental ranges. Fig. 3 shows the maximum run-up elevation of the water surface as the wave collided at $t=0.0 \mathrm{~s}$. In these plots, the amplitude $\varepsilon_{L}$ of the left-running wave varies as (i) 0.307 and (ii) 0.200 . The corresponding theoretical run-ups, calculated to the third order, agree well with the data obtained by the imaging technique.

Fig. 4 depicts a series of instantaneous velocity fields during a head-on collision, captured by the PIV method. The left, middle, and right panels show the fields of the left-running, compound, and 
right-running waves, respectively. The velocity vectors during the collision of two solitary waves were precisely determined in front of the video recording area. Until it collided with the oncoming wave, the solitary wave propagated as the underwater mass interacted with the stationary mass in the adjacent area. When the two amplitudes were almost equal $\left(\varepsilon_{R}=0.187\right.$ and $\left.\varepsilon_{L}=0.200\right)$, the patterns of the leftand right-running waves were complementary (see Fig. 4 (a)). At $t=-0.18 \mathrm{~s}$, the compound wave exhibited a quasi-symmetrical velocity pattern near the meeting site. We can verify that the merging wave surface accelerated toward its maximum level at $t=0.0 \mathrm{~s}$, when the kinetic and potential energies were maximally diminished and amplified, respectively. At this instant, the vertical velocity did not completely vanish because $\varepsilon_{R} \neq \varepsilon_{L}$. At $t=0.18 \mathrm{~s}$, the combined wave surface descended at high velocity between the right- and left-running wave crests. As the crests separated, the flow pattern of the colliding wave approached that of the superposed vectors of the right- and left-running waves. In this test, however, the vertical flow (down-welling) persisted over a larger area of the image (at $t=$ $0.36 \mathrm{~s}$ ). Fig. 4 (b) plots the PIV result for $\varepsilon_{R}=0.200$ and $\varepsilon_{L}=0.307$. The right- and left-running waves began interfering before reaching the test section. Prior to their meeting, the two solitary waves produced a rather irregular flow pattern. The water particles, which were not perfectly stationary at $t=$ $0.0 \mathrm{~s}$, thereafter increased their downward velocity and finally reduced their total speed. On careful inspection of the single and multiple wave velocity fields, we find that the interaction violated the quasi-linear superposition principle. Similar images for $\varepsilon_{R}=0.273$ and $\varepsilon_{L}=0.200$ are shown in Fig. 4 (c). The water particles were upwelled just ahead of each crest, generating a new crest of the colliding wave at $t=-0.18 \mathrm{~s}$. At $t=0.0 \mathrm{~s}$, the right-running wave exerted a non-negligible effect on the compound wave. At $t=0.18 \mathrm{~s}$, the water particles changed direction, moving downward in the space between the crests. The interaction between the two waves continued to drag out the vertical flow. The vertical velocity before and after the collision increased the kinetic energy of the colliding wave, with an inevitable effect on the phase delay. Fig. 5 compares the theoretical and experimental variations of the water surface in the range $-6.173 \leq t / \sqrt{h / g} \leq 6.173$. A perspective view of the changing water surface is presented in the range $-30.0 \leq x / h \leq 30.0$. The solid curve represents the water surface predicted by the third-order solution in the interval $\Delta t / \sqrt{h / g}=0.343$. Blue curves and red circles indicate the water surface at the first stage $(t / \sqrt{\mathrm{h} / \mathrm{g}}=-6.173)$ and the points along the water surface (determined by combined PMC and image thresholding), respectively. Fig. 5 (a) plots the water surface distribution during the collision of two smaller waves; $\varepsilon_{R}=0.187$ and $\varepsilon_{L}=0.200$. The theoretical curves accurately predict five phases of the measured profiles. From this consecutive record, we confirm that the experimental surface profiles support the phase-shift hypothesis after a collision. Fig. 5 (b) compares the theoretical and measured wave profiles for $\varepsilon_{R}=0.227$ and $\varepsilon_{L}=0.200$. The right- and left-running wave amplitudes behaved oppositely to the previous case, and unlike the collision between two counter-propagating solitary waves of equal amplitude, the compound wave profile is asymmetric. Once the pair of humps has disappeared, the profile becomes quasi-symmetrical at $t / \sqrt{h / g}=0.00$. Fig. 5 (c) displays the surface during the collision of two solitary waves with $\varepsilon_{R}=2.00$ and $\varepsilon_{L}=3.07$. In this case, the left-running wave was 1.5 times larger than the rightrunning wave. Both original waves began interacting before $t / \sqrt{h / g}=-6.143$, and continuously deformed thereafter. The nonlinear terms in the water-surface profile can no longer be ignored, because the wave interaction is highly nonlinear. Up to $t / \sqrt{h / g}=0.00$, the dimensionless local maxima of the measured colliding wave almost fit the third-order result; thereafter, the theory overestimates the experimental values. As the surface descended to its initial level, the two crests separated from the colliding wave and developed into two phase-shifted solitary waves. The phase shift was larger in the right-running wave than in the left-running wave, qualitatively consistent with the calculated phase shift. As the vertical distance of the physical test section was only $80 \mathrm{~cm}$, we could not expect to observe the asymptotic features; that is, the evolution of the colliding wave into two fully deformed waves. However, each profile can be distinguished with sufficient accuracy to warrant examination. Finally, we display the head-on collision of dual waves with dimensionless amplitudes of $\varepsilon_{R}=0.327$ and $\varepsilon_{L}=0.200$ (Fig. $5(\mathrm{~d})$ ). The total wave energy appears to be reduced after the collision between the two crests. The amplitude of the larger solitary wave was markedly reduced by energy transfer to the generated trailing wavetrain, as explained in the following analysis of the wave gauge experiments. 

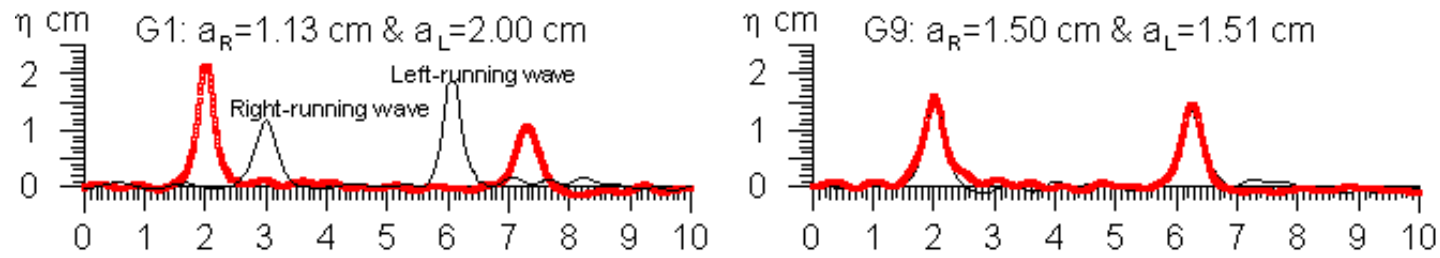

$\eta \mathrm{cm} \quad \mathrm{G} 2: \mathrm{a}_{\mathrm{R}}=1.23 \mathrm{~cm} \& \mathrm{a}_{\mathrm{L}}=2.02 \mathrm{~cm}$

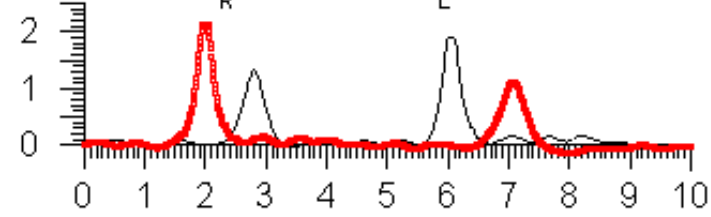

$\eta \mathrm{cm} G 10: a_{R}=1.60 \mathrm{~cm} \& a_{L}=1.51 \mathrm{~cm}$
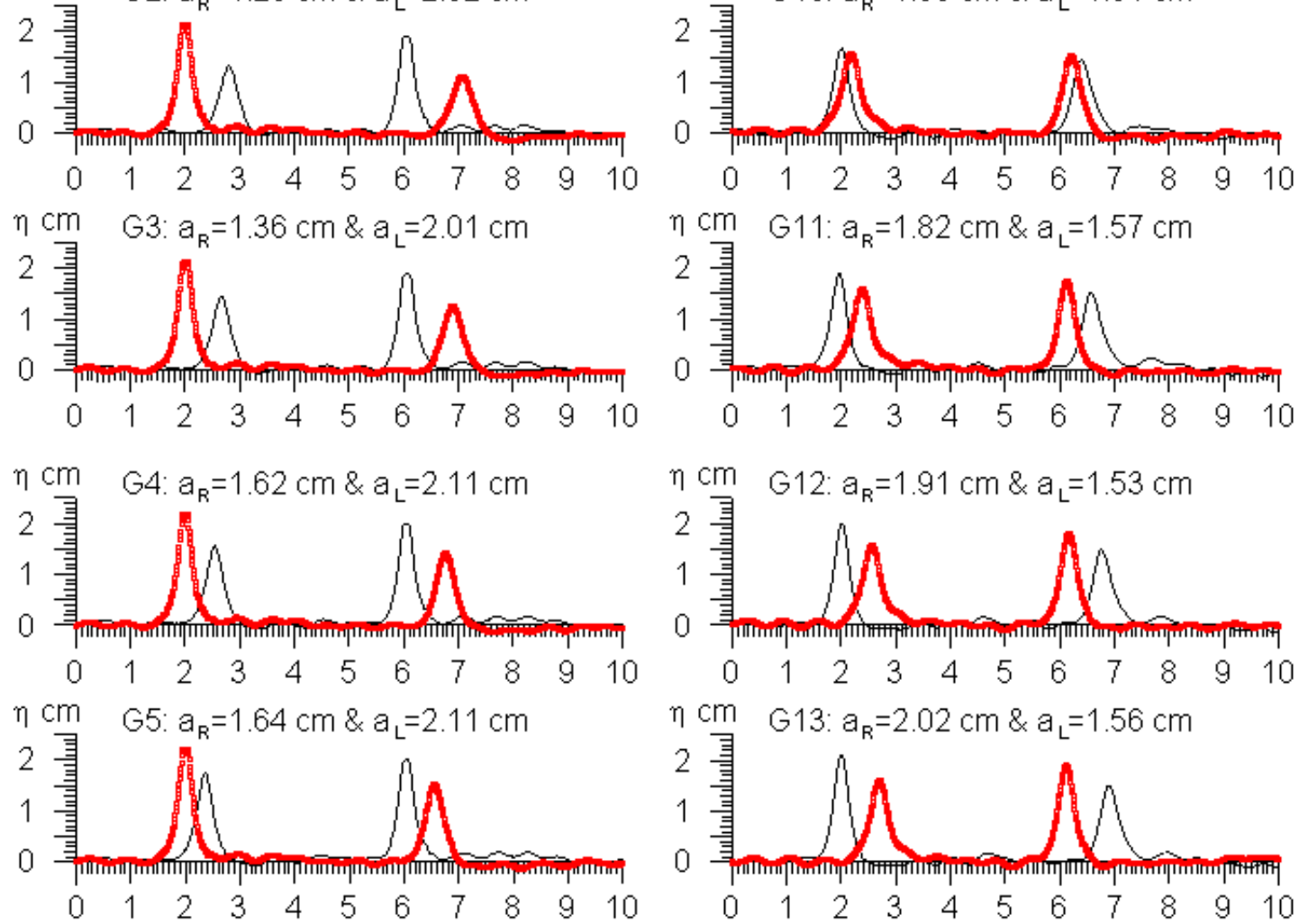

$\eta \mathrm{cm} G 13: \mathrm{a}_{\mathrm{R}}=2.02 \mathrm{~cm} \& \mathrm{a}_{\mathrm{L}}=1.56 \mathrm{~cm}$

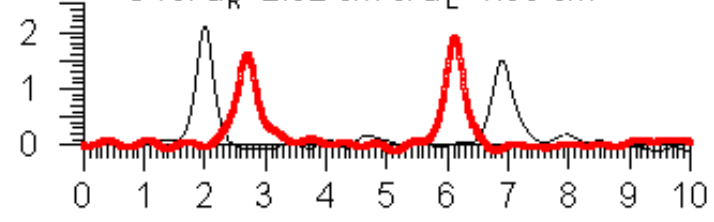

$\eta \mathrm{cm} \quad \mathrm{G} 6: \mathrm{a}_{\mathrm{R}}=1.74 \mathrm{~cm} \& \mathrm{a}_{\mathrm{L}}=2.15 \mathrm{~cm}$

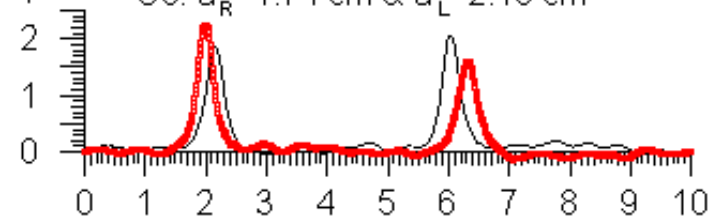

$\eta \mathrm{cm} \quad 14: \mathrm{a}_{\mathrm{R}}=2.08 \mathrm{~cm} \& \mathrm{a}_{\mathrm{L}}=1.56 \mathrm{~cm}$
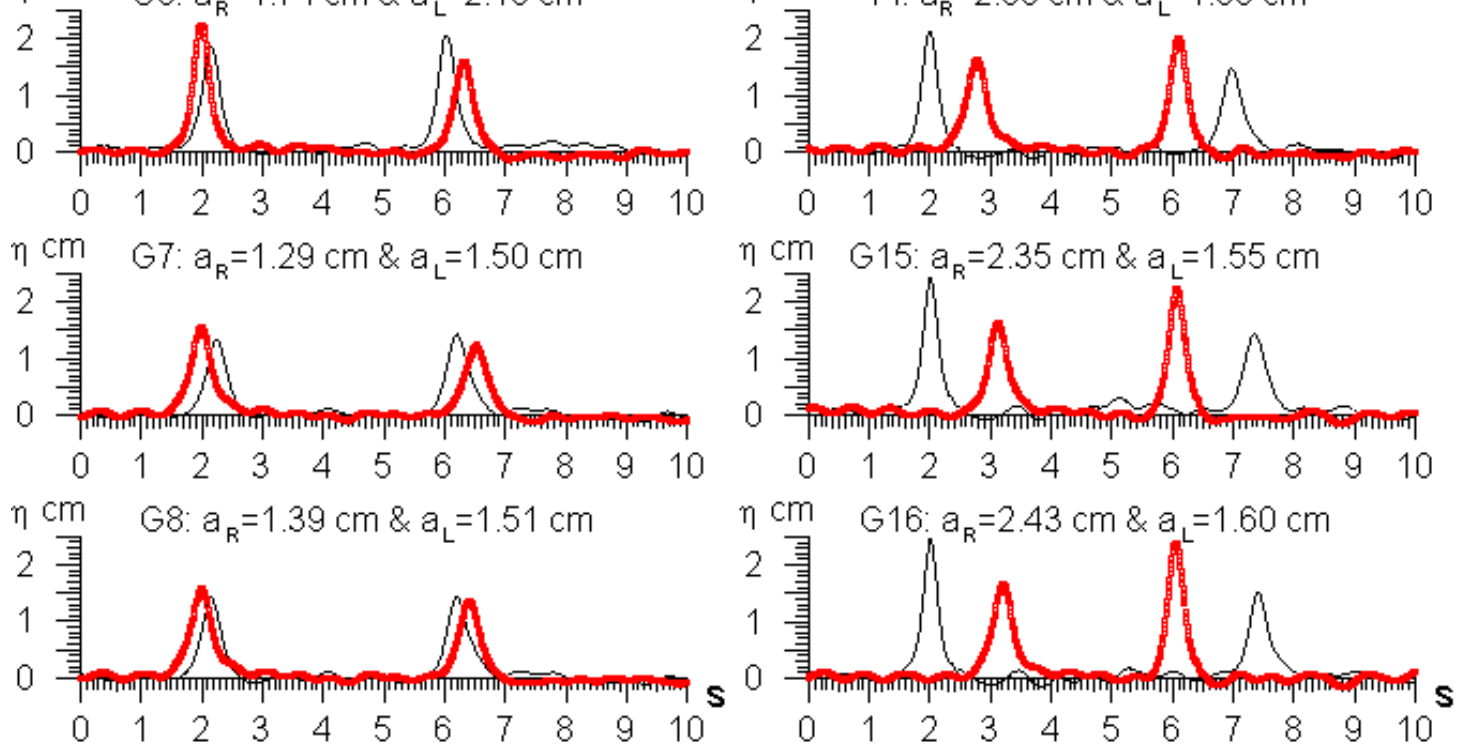

Fig. 6 Surface displacements from two gauges: (a) GAUGE-1 (black), and (b)GAUGE-2 (red). 

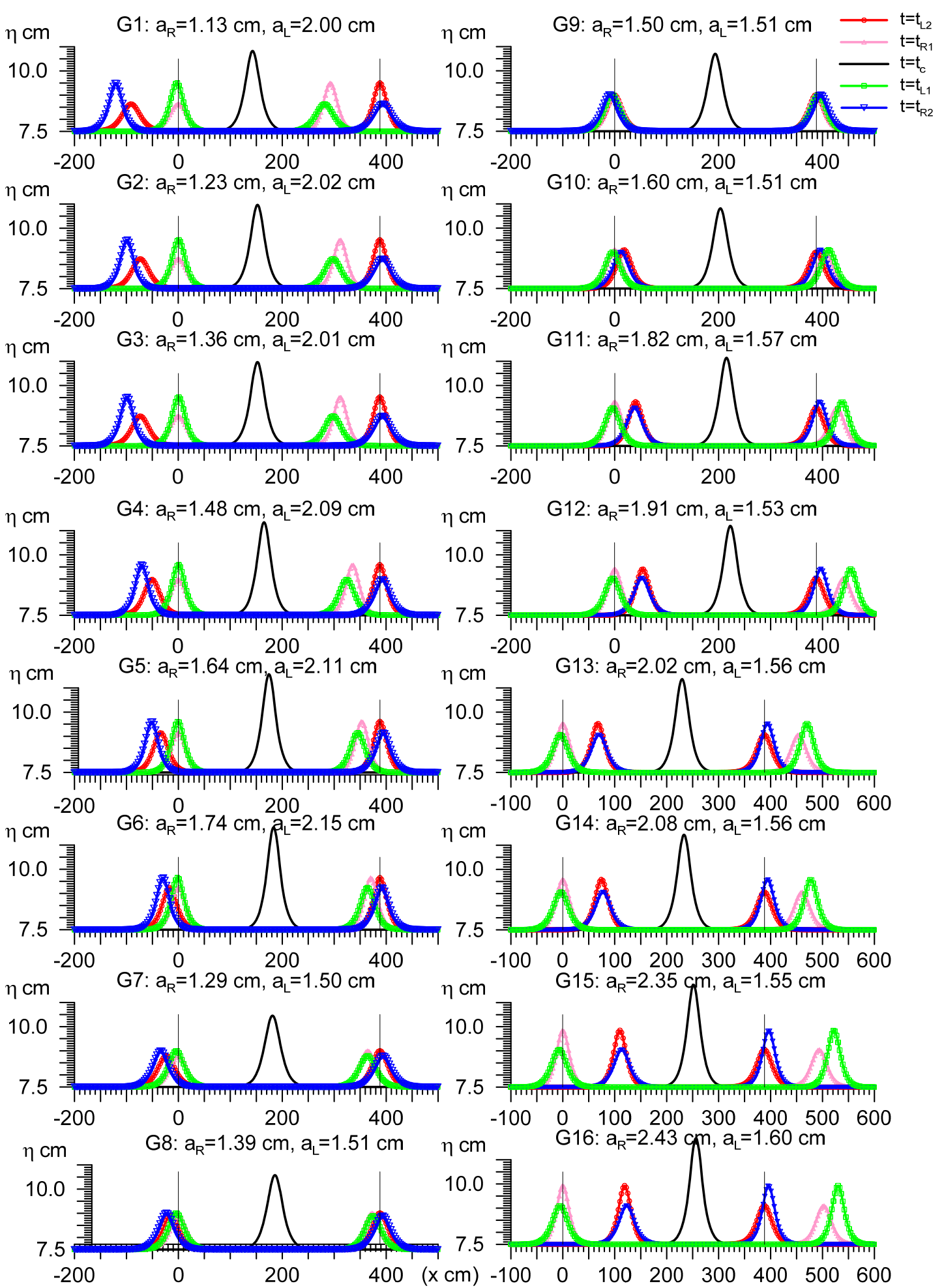

Fig. 7 Estimated spatial surface profiles in the collision process.

The second experiment was carried out to establish the validity of Eqs. (8)-(11). The surface data were measured by two wave gauges separated by $388 \mathrm{~cm}$ along the wave tank. This experiment quantified the asymptotic features of two interacting dual solitary waves with different amplitudes. To obtain the time interval of a wave crest from one gauge to another, we removed the transit moments before and after the interaction. Before generating two solitary waves, we tracked a single solitary wave propagating along the tank, traveling back and forth between the wave maker and the reflected 
wall. Fig. 6 presents the temporal surface profiles resulting from the dual wave measurements in tests G1-G16. In each panel, the trace develops into two humps, providing two local maxima that identify the crests. The right-running wave was reflected from the vertical wall, and interfered with the leftrunning wave. After the interaction, the left-running wave appeared at GAUGE-1. However, the rightrunning wave followed the left-running wave before its surface profile was measured by GAUGE- 2 . The amplitude of the interacted wave was $3-15 \%$ smaller than before the collision. Under these circumstances, both waves must reduce their speed.

Fig. 7 presents the analytically determined spatial surface displacements at five moments: (i) when the right-running wave crest passes GAUGE-1 $\left(t=t_{R 1}\right)$; (ii) when the left-running wave crest passes GAUGE-1 $\left(t=t_{L 1}\right)$; (iii) when the right-running wave reaches GAUGE-2 $\left(t=t_{R 2}\right)$; (iv) when the left-running wave reaches GAUGE-2 $\left(t=t_{L 2}\right)$; and (v) when both crests meet between the gauges $\left(t=t_{c}\right)$. These temporal order of these analyses is $t=t_{L 2}<t_{R 1}<t_{c}<t_{L 1}<t_{R 2}$ in cases G1-G9, and $t=t_{R 1}<t_{L 2}<t_{c}<t_{R 2}<t_{L 1}$ in cases G10-G16. In each case, the right and left vertical lines extended from the abscissa indicate the positions of GAUGE-1 and GAUGE-2, respectively. Assuming that the speeds of the right- and left-running waves in the preceding time domain are given by Eqs. (6) and (8), respectively, the maximum elevation occurs between $t=t_{R 1}$ and $t=t_{L 2}$. Fig. 8 is a three-dimensional plot of the theoretical phase shifts calculated by Eqs. (7) and (9), and the experimental phase shifts. The $x$ - and $y$-axes represent the dimensionless amplitudes of the target and oncoming waves respectively, and the z-axis denotes the phase shift. The red (green) bars indicate the phase shifts when the target and incoming waves are assigned as the left (right)- and right (left)-running waves, respectively. The experimental phase shifts were always larger than the theoretical ones in the red group, but the situation reversed in the green group. This result is consistent with higher overrun of the right-running (reflected) wave than the left-running wave.

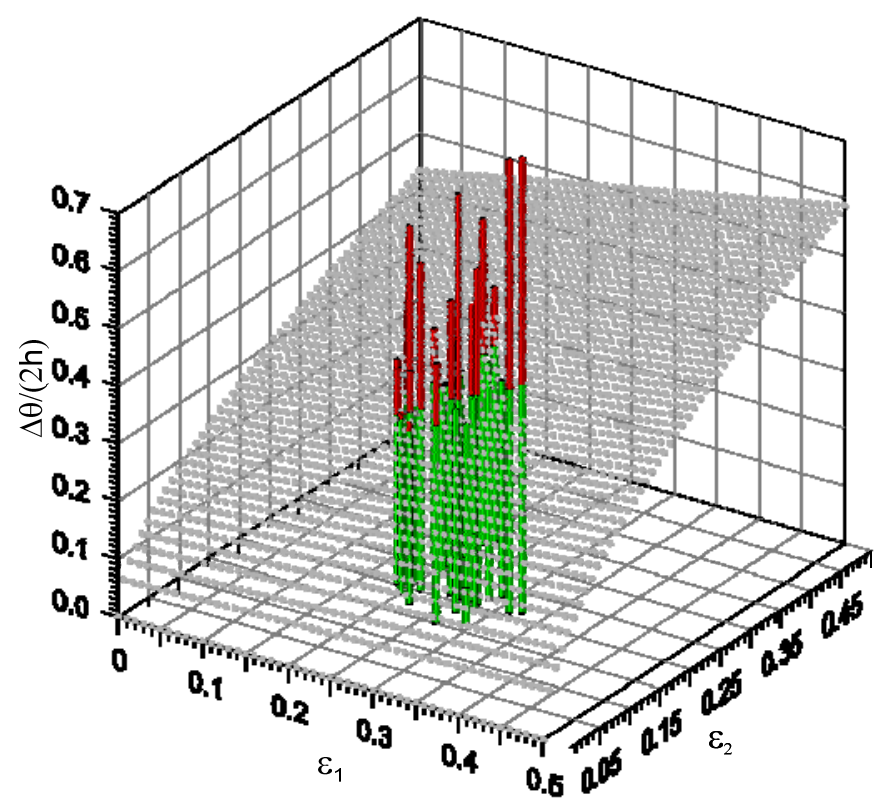

Fig.8 Phase sifts for original target and oncoming wave amplitudes after a collision occurred between two gauges.

\section{CONCLUSIONS}

We experimentally investigated the spatial velocity variations for two different kinds of dual solitary waves. The mechanics of the interaction between counter-propagating unequal solitary waves were studied by recent imaging techniques (the image thresholding method, PMC, and PIV). By adjusting the movement of the wave-generating paddle, we generated two waves that collided in the test section covered by the CCD camera. The hypothetical waves were assumed to merge into a colliding wave at $t \rightarrow 0$, and separate at $t \rightarrow+\infty$. The first wave reflected at the end of the wave tank and was returned to the wavemaker; the second was accurately timed to intercept the first at a prefixed 
location. The dynamics of the water surface were captured by the PMC and image thresholding methods. Nonlinear wave interactions are evident in Fig. 1, in which the initial wave crests are assumed to be largely separated at $t=-\infty$. In the measured surface profile, the maximum elevation during the collision of two crests exceeded the sum of both wave amplitudes. Our measured water surface displacements well matched the analytical solutions of Su and Mirie (1980). The images from the combined PMC and image thresholding method were consistent with our expectations; that nonlinear interactions induce phase shifts in the waves. A more careful temporal examination revealed that the compound wave develops phase shifts only after $t=0.0 \mathrm{~s}$. The separated waves were delayed relative to their expected arrival positions in the case of no interaction. From the PIV-measured velocity fields of the dual solitary and compound waves, we inspected the phase shift phenomenon in detail. Large accelerations were developed until the merging wave became maximally elevated; thereafter, the water surface largely decelerated as the compound wave split apart, and the solitary waves recovered their original profiles. In the velocity fields of the single and dual solitary waves, the compound wave exhibited strong nonlinear behavior during the head-on collision. To describe the general properties of the third-order compound wave, we measured the surface variations in the preceding and following time domains. Comparing the calculated and measured surface profiles, we found that the measured geometry, run-up, and phase lag were accurately predicted by the theory. In the wave gauge experiment, we accurately observed the wave speed, energy loss, and phase shift over a larger region than that covered by the PIV experiment. The gauge measurements revealed a weak wave train following the main wave in the same propagation direction. When the right-running wave was smaller (larger) in amplitude than the left-running wave, the smaller wave exhibited set-down (setup) of the free surface after the interaction. The experimentally determined wave speed was slightly below that predicted by third-order wave theory, and each wave recovered its original shape. The surface measurement provided a general impression of the irregular vibrations induced by accelerations and decelerations between both wave crests.

\section{REFERENCES}

Boussinesq, M.J. 1871. Théorie des ondes et des remous qui se propagent le long d'un canal réctangulaire horizontal, en communiquant au liquide contenu dans ce canal des vitesses sensiblement pareilles de la surface au fond, J. Math. Pures Appl., 17, 55-108.

Byatt-Smith, J.G.B. 1971. An integral equation for unsteady surface waves and a comment on the Boussinesq equation, J. Fluid Mech., 49, 625-633.

Byatt-Smith, J.G.B. 1988. The reflection of a solitary wave by a vertical wall, J. Fluid Mech., 197, 503-521.

Byatt-Smith, J.G.B. 1989. The head-on interaction of two solitary waves of unequal amplitude, J. Fluid Mech., 205, 573-579.

Chambarel, J., C. Kharif, and J. Touboul. 2009. Head-on collision of two solitary waves and residual falling jet formation, Nonlinear Process. Geophys. 16, 111-122.

Chen, Y. and H. Yeh. 2014. Laboratory experiments on counter-propagating collisions of solitary waves. Part 1. Wave interactions, J. Fluid Mech., 749, 577-596.

Constantin, A. 2011. Nonlinear water waves with applications to wave-current interactions and tsunamis, CBMS-NSF Regional Conference Series in Applied Mathematics, 81, 317p.

Drazin, P.G. and R.S. Johnson. 1989. Solitons: an Introduction, Cambridge, 226p.

Etoh, K. 1999. A study on particle identification in PIV - particle mask correlation method, Journal of Visualization, 1, 313-323.

Fermi, E., J. Pasta, and S.M. Ulam. 1955. Studies in nonlinear problems, Tech. Rep., LA-1940, Los Alamos Sci. Lab.

Gardner, C. S., J. M., Green, M. D., Kruskal, and R. M., Miura. 1967. Method for Solving the Korteweg-de Vries Equation, Phys. Rev. Lett. 19, 1095-1097.

Goring, D.G. 1979. Tsunamis -The propagation of long waves onto a shelf, Ph.D. thesis, Caltech, $337 \mathrm{p}$.

Hammack, J., D. Henderson, P. Guyenne and Y. Ming. 2004. Solitary-wave collisions, A Symp. to honor Theodore Yao-Tsu Wu, OMAE 2004, ASME, 1-12.

Johnson, R.S. 1997. A modern introduction to the mathematical theory of water waves, Cambridge, 445 p. 
Korteweg, D.J. and G. deVries. 1895. On the change of form of long waves advancing in a rectangular canal and on a new type of long stationary waves, Phil. Mag., 39, 422-443.

Maxworthy, T. 1976. Experiments on collisions between solitary waves, J. Fluid Mech., 76, 177-185.

Miles, J. W. 1977. Resonantly interacting solitary waves, J. Fluid Mech., 79, 171-179.

Mirie, R.M. and C.H. Su. 1982. Collisions between two solitary waves. Part 2. A numerical study, J. Fluid Mech., 115, 475-492.

Rayleigh, Lord. 1876. On waves, Phil. Mag. (5), 1, 257-279.

Renouard, D., F. Santos and A. Temperville. 1985. Experimental study of the generation, damping, and reflexion of a solitary wave, Dyn. Atmos. Oceans, 9, 341-358.

Russell, J.S. 1844. Report on waves, Rep. Meet. Brit. Assoc. Adv. Sci., 14, 311-390.

Russell, J.S. and J. Robison. 1837. Report on waves, Rep., Brit. Assoc. Adv. Sci., 417-496.

Su, C.H. and R.M. Mirie. 1980. On head-on collisions between two solitary wave, J. Fluid Mech., 98, 509-525.

Takehara, K., Adrian, R. J., Etoh, T., and Christensen, K. T. (2000). "A Kalman tracker for superresolution PIV." Exp. Fluids, 29(Supp. 1), S034-S41.

Umeyama, M. 2008. PIV techniques for velocity fields of internal waves over a slowly varying bottom topography, J. Water., Port, Coast. \& Oc. Eng., ASCE, 134(5), 286-298.

Umeyama, M. 2011. Coupled PIV and PTV measurements of particle velocities and trajectories for surface waves following a steady current, J. Water., Port, Coast. \& Oc. Eng., ASCE, 137(2), 85-94.

Umeyama, M. 2012. Eulerian/Lagrangian analysis for particle velocities and trajectories in a pure wave motion using particle image velocimetry, In a Theme Issue 'Nonlinear Water Waves' edited by Adrian Constantin, Phil. Trans. Roy. Soc. A, Roy. Soc. Pub., 370(1964), 1687-1702.

Umeyama, M. 2013. Investigation of single and multiple solitary waves using superresolution PIV, J. Water., Port, Coast. \& Oc. Eng., ASCE, 139(4), 303-313.

Umeyama, M., N. Ishikawa and R. Kobayashi. 2014. High-resolution PIV measurements for rear-end and head-on collisions of two solitary waves, Proc., ICCE, ASCE, 34, Waves.40.

Umeyama, M., T. Shintani and S. Watanabe. 2010. Measurements of particle velocities and trajectories in a wave-current motion using PIV and PTV, Proc., ICCE, ASCE, 32, Waves.2.

Zabusky, N.J. and M.D. Kruskal. 1965. Interaction of solitons in a collisionless plasma and the recurrence of initial states, Phys. Rev. Lett., 15, 240-3. 